\title{
Margatoxin mitigates CCl4-induced hepatic fibrosis in mice via macrophage polarization, cytokine secretion and STAT signaling
}

\author{
BAO-MING WU ${ }^{1-4^{*}}$, JUN-DA LIU ${ }^{1-5 *}$, YUAN-HAI LI ${ }^{1-5}$ and JUN LI ${ }^{1-4}$ \\ ${ }^{1}$ School of Pharmacy, Anhui Key Laboratory of Bioactivity of Natural Products, Anhui Medical University; \\ ${ }^{2}$ The Key Laboratory of Anti-Inflammatory and Immune Medicine, Anhui Medical University, Ministry of Education; \\ ${ }^{3}$ Institute for Liver Diseases of Anhui Medical University; ${ }^{4}$ Anhui Institute of Innovative Drugs, Anhui Medical University; \\ ${ }^{5}$ The First Affiliated Hospital of Anhui Medical University, Hefei, Anhui 230032, P.R. China
}

Received May 2, 2019; Accepted October 14, 2019

DOI: 10.3892/ijmm.2019.4395

\begin{abstract}
A number of macrophage phenotypes have been previously identified as crucial regulators in the progression of hepatic fibrosis (HF). Cytokines from macrophages or Kupffer cells (KCs) have also been identified to be important regulators in HF. Blocking Kv1.3 in models of $\mathrm{HF}$, regulating macrophage polarization and cytokine secretion have not yet been assessed as potential treatments options for this condition. In the current study, a model of carbon tetrachloride $(\mathrm{CCl} 4)$-induced $\mathrm{HF}$ was established and examined the effects of margatoxin (MgTX; an inhibitor of Kv1.3) on HF. Hematoxylin and eosin, Masson's trichrome and immunohistochemistry staining were performed to determine whether MgTX can alleviate liver fibrosis. To elucidate the mechanisms through which MgTX attenuates liver injury, reverse transcription-quantitative PCR and western blot analysis were used to detect polarized macrophage markers in RAW264.7 cells and cytokines were examined using ELISA. Furthermore, macrophage polarization signal transducer and activator of transcription (STAT) signaling, which is associated with macrophage polarization, was identified in RAW264.7 cells. The results revealed that MgTX protected the mice from CCl4-induced liver fibrosis. Furthermore, MgTX decreased the expression of M1 phenotype biomarkers, and increased the expression of M2 phenotype biomarkers in CCl4-induced HF. Additionally, the production of pro-inflammatory cytokines
\end{abstract}

Correspondence to: Professor Jun Li, School of Pharmacy, Anhui Key Laboratory of Bioactivity of Natural Products, Anhui Medical University, 81 Meishan Road, Hefei, Anhui 230032, P.R. China E-mail:1j@ahmu.edu.cn

Professor Yuan-Hai Li, The First Affiliated Hospital of Anhui Medical University, 218 Jixi Road, Hefei, Anhui 230032, P.R. China E-mail: liyuanhai-1@163.com

*Contributed equally

Key words: hepatic fibrosis, margatoxin, macrophages polarization, cytokines was decreased and interleukin-10 production was increased in the serum of mice with HF injected with MgTX. Furthermore, MgTX was found to regulate the expression of M1 markers by suppressing p-STAT1 activity and increasing the expression of M2 markers by promoting p-STAT6 activity. On the whole, the findings of this study demonstrate that MgTX is able to alleviate $\mathrm{CCl} 4$-induced $\mathrm{HF}$ in mice, possibly via macrophage polarization, cytokine secretion and STAT signaling.

\section{Introduction}

Fibrosis is a protective reaction that is activated in response to hepatic injury, causing a variety of diseases that result in hepatocellular death. Therefore, liver fibrosis is observed in patients with chronic viral hepatitis, non-alcoholic fatty liver disease, alcoholic liver disease, obesity, cholestatic and autoimmune liver diseases $(1,2)$. Previous studies have indicated that the inflammatory response in the liver plays a crucial role in hepatic fibrogenesis during hepatic fibrosis (HF) (3). Hepatic macrophages, or Kupffer cells (KCs), are important immune cells that are associated with the pathogenesis of chronic liver injury, and have been recognized as potential targets for use in the treatment of fibrosis (4). Liver fibrosis has been widely demonstrated to be a reversible process. Hepatic macrophages can serve dual functions in the progression of experimental hepatic fibrosis, and can reverse mechanisms that are associated with the degradation of excessive extracellular matrix deposition in the liver (5). The investigation into novel compounds that can be used to regulate macrophage function is required for the identification of a therapeutic strategy for HF.

Carbon tetrachloride (CCl4)-induced HF is characterized by the activation of $\mathrm{KCs}$ and the relevant immune response, which results in the secretion of cytokines, chemokines and other pro-inflammatory factors (6). The mechanisms underlying the role of macrophages in HF have not been fully elucidated. Macrophages are classified into two phenotypes, those with M1 type pro-inflammatory function and those with M2 type-immunoregulatory function $(7,8)$. Different phenotypes of macrophages have been identified to carry out different functions in the progression of HF. M1 macrophages are considered to induce pro-inflammatory cytokines and fibrogenesis, while M2 macrophages can be subdivided into 
at least 5 subtypes; however, their function in inflammation and fibrosis remains undetermined (9). Previous studies have demonstrated that a variety of M2 subtypes may exhibit pro- or anti-fibrotic activity (10-13). The mechanisms of macrophages as regards the regulation of liver fibrosis are associated with macrophage polarization. A variety of macrophage subtypes secrete a number of different cytokines, and this can result in a dual function response during the progression of HF.

The current study aimed to determine the effects of margatoxin (MgTX) on the polarization of macrophages in RAW264.7 cells, and to detect the serum levels of inflammatory cytokines following MgTX treatment in a model of $\mathrm{HF}$. Hepatic macrophages in the liver, which are also known as $\mathrm{KCs}$, when activated during an inflammatory condition, result in the release of a number of pro-inflammatory cytokines and chemokines, and an increase the activation of hepatic stellate cells (HSCs) (14). M1 macrophages are classical macrophages that exhibit antiviral activity and release pro-inflammatory cytokines, including tumor necrosis factor- $\alpha$ (TNF- $\alpha$ ), interleukin (IL)-1 $\beta$ and reactive oxygen species, while M2 macrophages are activated macrophages that promote the defense against parasitic infections and are associated with tissue remodeling, and secrete immune-modulatory cytokines, including IL-10, transforming growth factor- $\beta$ (TGF- $\beta$ ), IL-4 and IL-13 (15). Increasing evidence has indicated that a number of pro-inflammatory cytokines, including TNF- $\alpha$ and TGF- $\beta$, play a key role in the initiation of stellate cell activation. In other liver diseases, fibrosis has been indicated to be associated with the production of M2-associated cytokines and TGF- $\beta$ (15). However, whether these cytokines originate from M2 macrophages during the modulation of extracellular matrix remodeling remains undetermined. Previous studies had revealed that M2 macrophage-derived cytokines, including IL-4, IL-13 and IL-10, regulate the chronic inflammatory response and ameliorate wound healing in the liver (16-19). On the whole, different macrophage subtypes secrete a number of different cytokines, which play key roles in the progression of HF.

$\mathrm{Kv1.3}$ is a voltage-gated potassium channel subtype, which has 6 transmembrane domains, including a voltage sensor and a pore-forming region (20). MgTX is a 39 amino-acid-long peptide that is stabilized by 3 disulfide bridges, has a molecular weight of 4,185 and is isolated from the venom of the scorpion Centruroidesn margaritatus. MgTX exhibits a high affinity for Kv1.3 and is a selective inhibitor of the Kv1.3 channel. Kv1.3-selective blockers have been indicated to be effective in reducing disease severity in a number of animal models of autoimmunity (21-24). Previous studies have demonstrated that the selective Kv1.3-channel inhibition can significantly suppress the number of leukocytes, and the progression of renal fibrosis exhibits a significant decrease in cortical cell cycle marker expression. Active Kv1.3 channels can promote cardiac fibrosis by secreting TGF- $\beta$. The blockade of voltage-dependent potassium channels (Kv) by specific antagonists decreases macrophage cytokine production and inhibits proliferation (25-27). However, whether MgTX is associated with HF has not, to the best of our knowledge, yet been determined. The current study aimed to assess whether MgTX can ameliorate HF in vivo, and to explore the underlying mechanisms that govern the association between Kv1.3 and macrophages polarization and cytokine secretion.

\section{Materials and methods}

Mouse model of HF. All animal protocols were approved by the Animal Care and Use Committee of Anhui Medical University, China. Male C57BL/6 mice (age, 4-6 weeks) were obtained from the Experimental Animal Center of Anhui Medical University for use in creating the model of CCl4-induced liver fibrosis. Early HF was generated by a bi-weekly intraperitoneal injection of CCl4 (20\% in olive oil) at a dose of $0.01 \mathrm{ml} / \mathrm{g} / \mathrm{mouse}$ for 4 weeks. The control mice were treated intraperitoneally with the same volume of olive oil at the same time intervals. MgTX was diluted in PBS and injected at $1 \mathrm{ml}$ by intraperitoneal injection at 3 different doses $1 \mathrm{~h}$ prior to the $\mathrm{CCl} 4$ (olive oil) injection, namely the doses of MgTX used were $0.4179 \mathrm{mg} / \mathrm{kg}$ (high, H), $0.139 \mathrm{mg} / \mathrm{kg}$ (moderate, M), $0.0418 \mathrm{mg} / \mathrm{kg}$ (low, L).

Collection of mouse blood and tissues. Blood samples were collected from orbital sinus by rapidly removing the eyeball after the mice were anesthetized with chloral hydrate $(4 \%, 400 \mathrm{mg} / \mathrm{kg})$ by intraperitoneal injection. Approximately $1 \mathrm{ml}$ blood was collected in an EP tube for each mouse. Following blood sample collection, the mice were sacrificed by cervical dislocation. Liver tissues were obtained by a midline laparotomy. Liver tissues were either fixed in $4 \%$ formalin for immunohistochemistry, or stored at $-80^{\circ} \mathrm{C}$ for use in western blot analysis. All animal studies were conducted following the approval from the Institutional Animal Care and Use Committee.

Histopathology and immunohistochemical staining. The central left hepatic lobe was sectioned and fixed in $4 \%$ paraformaldehyde for $48 \mathrm{~h}$. Tissues were then embedded in paraffin, and 5- $\mu \mathrm{m}$-thick slices were stained using hematoxylin (BA-4041, Baso) and eosin (BA-4022, Baso) for morphological analysis, stained with hematoxylin for $20 \mathrm{~min}$ and eosin for $1 \mathrm{~min}$ at room temperature. Masson's trichrome and Sirius red staining were used for the evaluation of collagen expression. Masson's trichrome kit (MST-8003/8004, Maixin-Bio) and Sirius red staining kit (36324ES60, Yeasen Biotechnology (Shanghai) Co., Ltd.) were carried out according to the manufacturer's instructions at room temperature. Immunostaining was performed on paraffin-embedded materials. The following primary antibodies were used: Matrix metalloproteinase (MMP)12 rabbit polyclonal (diluted 1:100; 22989-1-AP, Proteintech), MMP13 rabbit polyclonal (diluted 1:200; bs-10581R, Bioss), periostin (POSTN) rabbit polyclonal (diluted 1:200; bs-4994R, Bioss), C-C motif chemokine ligand 2 (CCL2) rabbit polyclonal (diluted 1:100; bs-1101R, Bioss), Kv1.3 rabbit polyclonal (diluted 1:100; bs-10229R, Bioss), $\delta$-catenin rabbit polyclonal (diluted 1:200; bs-7000R, Bioss), $\alpha$-SMA rabbit polyclonal (diluted 1:200; ab5694, Abcam), collagen I rabbit polyclonal (diluted 1:150; bs-10423R, Bioss) were used; the primary antibody reactions were incubated for $30 \mathrm{~min}$ at room temperature. A secondary antibody kit (PV-6000, Zsbio) was used for immunohistochemical staining. The average optical density for the semi-quantitative analysis of immunohistochemical staining was performed by CaseViewer (pananoramic MIDI, 3DHISTECH) and JD801.

ELISA. Serum was extracted from blood by centrifugation $(-1,000 \mathrm{x} \mathrm{g}, 10 \mathrm{~min})$ at $4^{\circ} \mathrm{C}$ and stored at $-80^{\circ} \mathrm{C}$ for subsequent use. 
Table I. Sequences of primers used for RT-qPCR.

\begin{tabular}{llcll}
\hline & Gene & Amplicon size $(\mathrm{bp})$ & \multicolumn{1}{c}{ Forward primer $\left(5^{\prime} \rightarrow 3^{\prime}\right)$} & \multicolumn{1}{c}{ Reverse primer $\left(5^{\prime} \rightarrow 3^{\prime}\right)$} \\
\hline Mouse & $\beta$-actin & 120 & AGTGTGACGTTGACATCCGT & TGCTAGGAGCCAGAGCAGTA \\
& CCL-2 & 128 & AACTGCATCTGCCCTAAGGT & CTGTCACACTGGTCACTCCT \\
Mrc2 & 83 & ACGACTGTGAGACCTTCTGG & CCTCCAGGACAGTGTGGATT \\
IL-10 & 88 & TGCACTACCAAAGCCACAAG & TCAGTAAGAGCAGGCAGCAT \\
Arg-1 & 135 & GCAGTTGGAAGCATCTCTGG & GAGAAAGGACACAGGTTGCC \\
TNF- $\alpha$ & 133 & GACAGTGACCTGGACTGTGG & TGAGACAGAGGCAACCTGAC \\
IL-1 $\beta$ & 98 & GAAGAAGAGCCCATCCTCTG & TCATCTCGGAGCCTGTAGTG \\
CD163 & 94 & ATGGGTGGACACAGAATGGT & AGCTCACAGCCACAACAAAG \\
iNOS & 143 & CCTTGTTCAGCTACGCCTTC & CTTCAGAGTCTGCCCATTGC
\end{tabular}

The levels of IL-1 $\beta$, IL-6, IL-10, IL-20, TNF- $\alpha$ and TGF- $\beta$ were determined using mouse serum using ELISA kits according to the manufacturer's protocol. A mouse IL-1 $\beta$ ELISA kit (cat. no. RK00006), mouse IL-6 ELISA kit (cat. no. RK00008), mouse IL-10ELISA kit(cat.no.RK00016), mouse IL-20ELISAkit (cat. no. RK00110), mouse TNF- $\alpha$ ELISA kit (cat. no. RK00027) and a mouse TGF- $\beta 1$ ELISA kit (cat. no. RK00057) were used and were all purchased from ABclonal, Inc.

Cells and cell culture. RAW264.7 cells were purchased from the Type Culture Collection of the Chinese Academy of Sciences. The cells were cultured in DMEM (Gibco; Thermo Fisher Scientific, Inc.) supplemented with FBS (10\%; Gibco; Thermo Fisher Scientific, Inc.), penicillin and streptomycin $(1 \%)$, and cultured at $37^{\circ} \mathrm{C}$ in a $5 \% \mathrm{CO}_{2}$ incubator. The RAW264.7 cells were treated with lipopolysaccharide LPS $(1 \mu \mathrm{g} / \mathrm{ml})$ combined with interferon (IFN)- $\gamma(10 \mathrm{ng} / \mathrm{ml})$ for $24 \mathrm{~h}$ to polarize the M1 macrophage phenotype, or were treated with IL-4 $(15 \mathrm{ng} / \mathrm{ml})$ for $24 \mathrm{~h}$ to induce the M2 macrophage phenotype. The RAW264.7 cells were also treated with $\operatorname{MgTX}(10 \mathrm{nM})$ for $24 \mathrm{~h}$ at $37^{\circ} \mathrm{C}$, as previously described (27).

Total RNA extraction and reverse transcription-quantitative $P C R(R T-q P C R)$. Total RNA was extracted from the RAW264.7 cell lines using TRIzol ${ }^{\circledR}$ reagent (Invitrogen; Thermo Fisher Scientific, Inc.). A ThermoScript RT-qPCR synthesis kit (Fermentas; Thermo Fisher Scientific, Inc.) was used to synthesize the cDNA according to the manufacturer's protocol. RT-qPCR-was performed using ThermoScript RT-qPCR kits (Fermentas; Thermo Fisher Scientific, Inc.). $\beta$-actin was used for normalization. Relative RNA expression was calculated using the standard $2^{-\Delta \Delta \mathrm{Cq}}$ method (28). Each experiment was performed in triplicate and repeated at least 3 times. The sequences of the primers $\left(5^{\prime}-3^{\prime}\right)$ are presented in Table I.

Western blot analysis. The RAW264.7 cells were lysed using protein extraction solution (Beyotime Institute of Biotechnology). The protein concentration of each sample was measured using a NanoDrop 2000 Spectrophotometer (Thermo Fisher Scientific, Inc.). Samples (20 $\mu \mathrm{g} /$ lane) underwent SDS-PAGE (10\%) and were transferred onto PVDF membranes (EMD Millipore). The PVDF membranes were blocked with $5 \%$ non-fat milk for $2 \mathrm{~h}$, and subsequently incubated with primary antibodies at $4^{\circ} \mathrm{C}$ overnight. Primary antibodies against $\delta$-catenin (1:300, cat. no. bs-7000R, Bioss), $\alpha$-SMA (1:300, cat. no. bs-0189R, Bioss), collagen I (1:300, cat. no. bs-10423R, Bioss), MMP12 (1:600, cat. no. 22989-1-AP, Proteintech), MMP13 (1:300, cat. no. bs-10581R, Bioss), POSTN (1:300, cat. no. bs-4994R, Bioss), CCL2 (1:500, cat. no. bs1101R, Bioss), Kv1.3 (1:300, cat. no. bs-10229R, Bioss), iNOS (1:1,000, cat. no. ab4999, Abcam), TNF- $\alpha$ (1:500, cat. no. BS1857, Bioworld), IL- $\beta$ (1:300, cat. no. bs0812R, Bioss), IL-10 (1:1,000, cat. no. bs0698R, Bioss), CD163 (1:300, cat. no. bs2527R, Bioss), Arg-1 (1:200, cat. no. sc-47715, Santa Cruz Biotechnology), Mrc2 (1:500, cat. no. ab70132, Abcam) and $\beta$-actin (1:500, cat. no. TA-09, Zs-BIO) were used. Subsequently, the PVDF membranes were washed 3 times with TBST, followed by incubation with secondary antibodies [HRP-goat anti-rabbit IgG (1:1,000; cat. no. ZB-2305; Zs-BIO)] at room temperature for $1.5 \mathrm{~h}$. After washing 3 times with TBST, the protein blots were observed using an ECL-chemiluminescent kit (ECL-plus; Thermo Fisher Scientific, Inc.), and the gray values of the bands were measured using ImageJ software (version 1.4.3.67).

Statistical analysis. Statistical analysis was performed using the Statistical Package for Social Sciences v.13.0 (SPSS, Inc.). Data are summarized from at least 3 experiments and are presented as the means \pm standard deviation. The statistical evaluation of the results was performed using a one-way ANOVA. Multiple comparisons between the groups were performed using the SNK method. A value of $\mathrm{P}<0.05$ was considered to indicate a statistically significant difference.

\section{Results}

Kv1.3 inhibition alleviates CCl4-induced liver fibrosis in mice. To investigate the degree of HF in C57BL/6J mice with CCl4-induced HF, histopathological analysis was performed. Hematoxylin and eosin staining, Masson's staining and Sirius Red staining demonstrated that the CCl4 (20\%) injection caused significant liver fibrosis, including serious steatosis, inflammatory cell infiltration and fibrosis in the main block of mouse livers compared with the control group. In comparison, no evidence of liver injury was observed in the control group with/without MgTX $(0.4179 \mathrm{mg} / \mathrm{kg})$. MgTX was therefore indicated to attenuate liver fibrosis in mice with CCl4-induced HF (Fig. 1A). Immunohistochemistry from the liver fibrosis tissues 
A

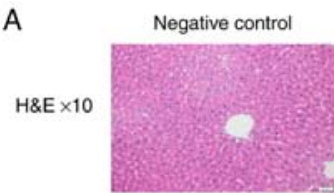

H\&E $\times 20$

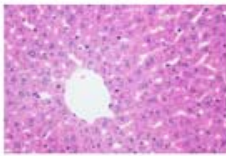

Masson's

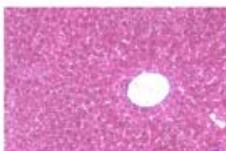

Sirius red

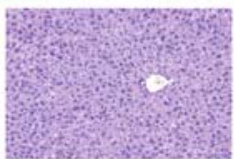

B
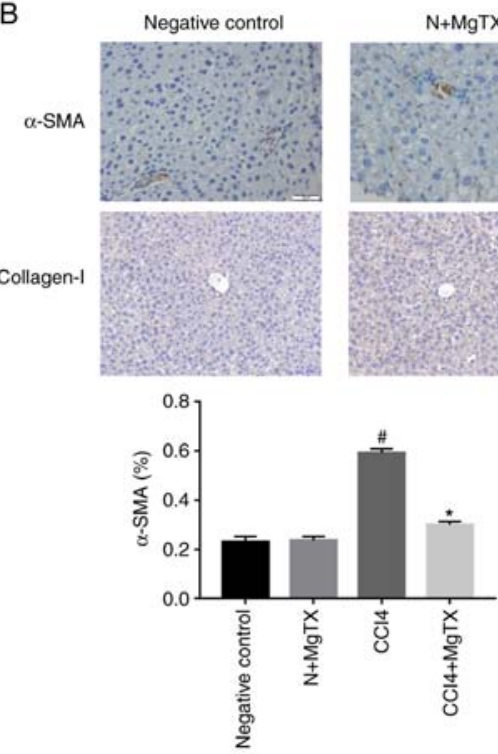

C
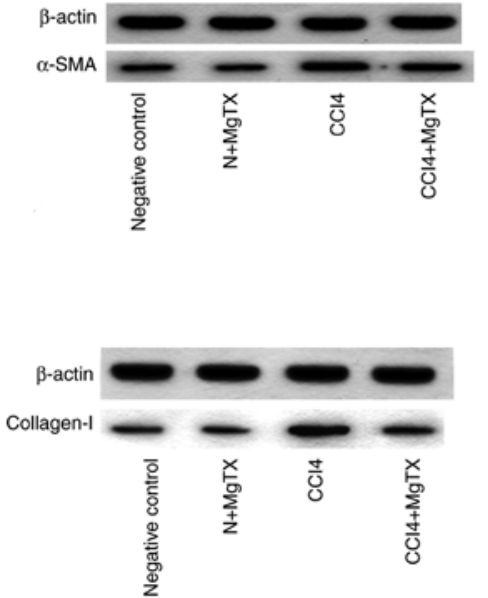

$\mathrm{N}+\mathrm{MgTX}$
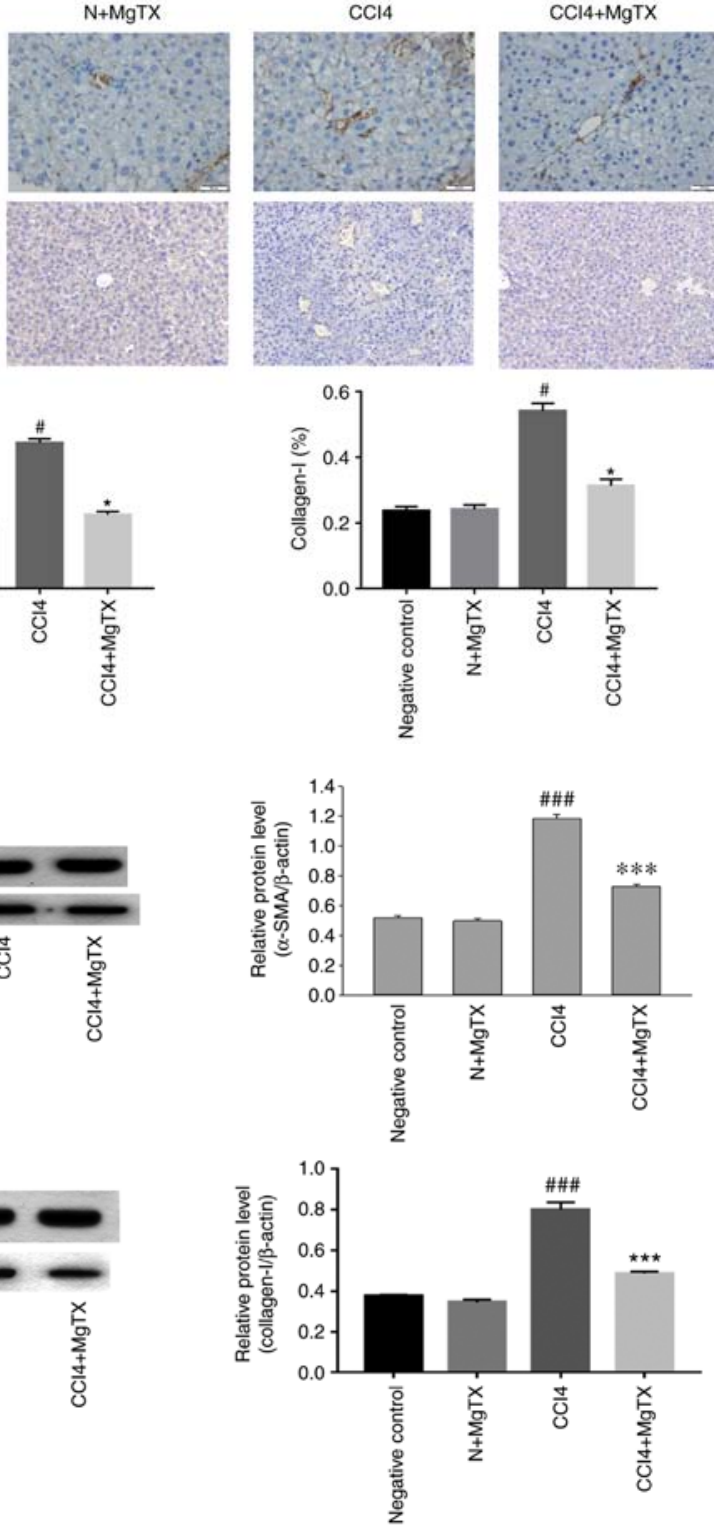

Figure 1. MgTX (0.4179 mg/kg) ameliorates CCl4-induced HF in C57BL/6J mice. (A) Representative hematoxylin and eosin staining, Masson's staining and Sirius Red staining of liver tissues indicated an incomplete hepatic lobule structure and irregularly arranged hepatic plates. Scattered degeneration, necrosis and numerous inflammatory cells immersed in liver tissue were identified compared with the HF model group treated with MgTX (magnification, x200; black arrows, fibrosis; green arrows, steatosis; red arrows, inflammatory cell infiltration). (B) Immunohistochemistry and (C) western blot analysis of hepatic fibrosis tissues revealed increased $\alpha$-SMA and collagen I expression compared with the CCl4-exposed group treated with MgTX. ${ }^{*} \mathrm{P}<0.05,{ }^{* * * *} \mathrm{P}<0.001$ vs. CCl4; ${ }^{\#} \mathrm{P}<0.05$, ${ }^{\# \#} \mathrm{P}<0.001$ vs. negative control. Representative views from each group are presented (original magnification, x20; $\mathrm{n}=6$ ). MgTX, margatoxin; $\mathrm{CCl} 4$, carbon tetrachloride; $\mathrm{HF}$, hepatic fibrosis. 

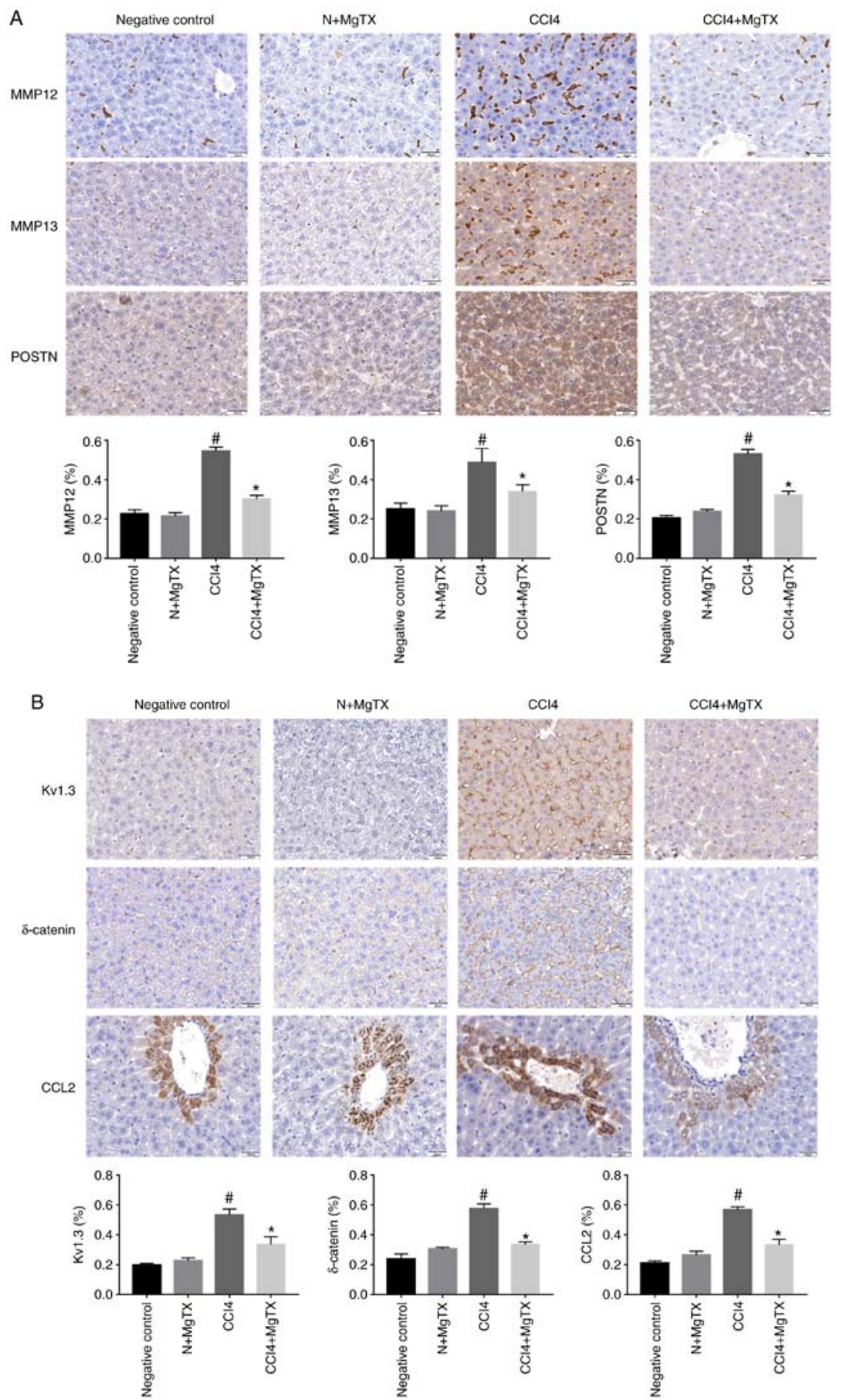

$\mathrm{N}+\mathrm{MgTX}$

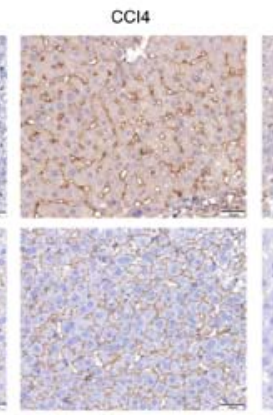

CCI4+MgTX

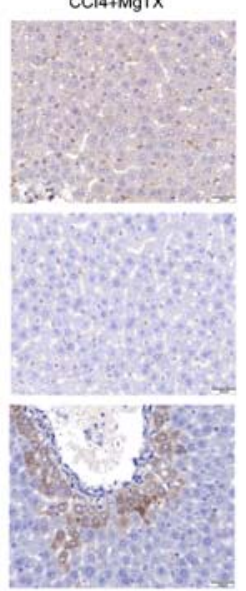

Figure 2. MgTX (0.4179 mg/kg) decreases the expression of HF-associated proteins in C57BL/6J mice with CCl4-induced HF (x200 field). (A) MMP12, MM13 and POSTN were associated with the amount of ECM and collagen. Immunohistochemistry of liver fibrosis tissues revealed MMP12, MM13 and POSTN exhibited increased staining compared with the HF group treated with MgTX. "P<0.05, CCl4 vs. CCl4 + MgTX group; " $\mathrm{P}<0.05$, CCl4 vs. N group. (B) CCL2, Kv1.3 and $\delta$-catenin are associated with the migration of macrophages. Immunohistochemistry of liver fibrosis tissues revealed increased staining for CCL2 and Kv1.3 compared with the HF group treated with MgTX. "P<0.05, CCL4 vs. CCL4 + MgTX group; ${ }^{\text {P }}<0.05$, CCL4 vs. N group. n=6. MgTX, margatoxin; HF, hepatic fibrosis; CCl4, carbon tetrachloride; CCL2, C-C motif chemokine ligand 2; MMP, matrix metalloproteinase; POSTN, periostin; ECM, extracellular matrix.

revealed extensive $\alpha$-SMA- and collagen I-positive staining, whereas the liver tissues in the control group with/without MgTX indicated decreased staining. Additionally, the results of western blot analysis for $\alpha$-SMA and collagen I protein expression were consistent with the results from immunohistochemistry (Fig. 1B and C). These results indicated that the mouse model of CCL4-induced liver fibrosis was successfully established and that MgTX protected the mice from CCl4-induced HF.
MgTX decreases the expression of HF-associated proteins in mice. To investigate the expression levels of proteins that are associated with HF in mice following the MgTX injection, immunohistochemistry and western blot analysis were performed to determine the expression of MMP12, MMP13, POSTN, $\delta$-catenin, Kv1.3 and CCL2. Immunohistochemical analysis of the liver tissues revealed that all proteins in the MgTX-treated mice with HF were decreased compared with the tissues from the mice with CCl4-induced HF not treated with MgTX (Fig. 2). 

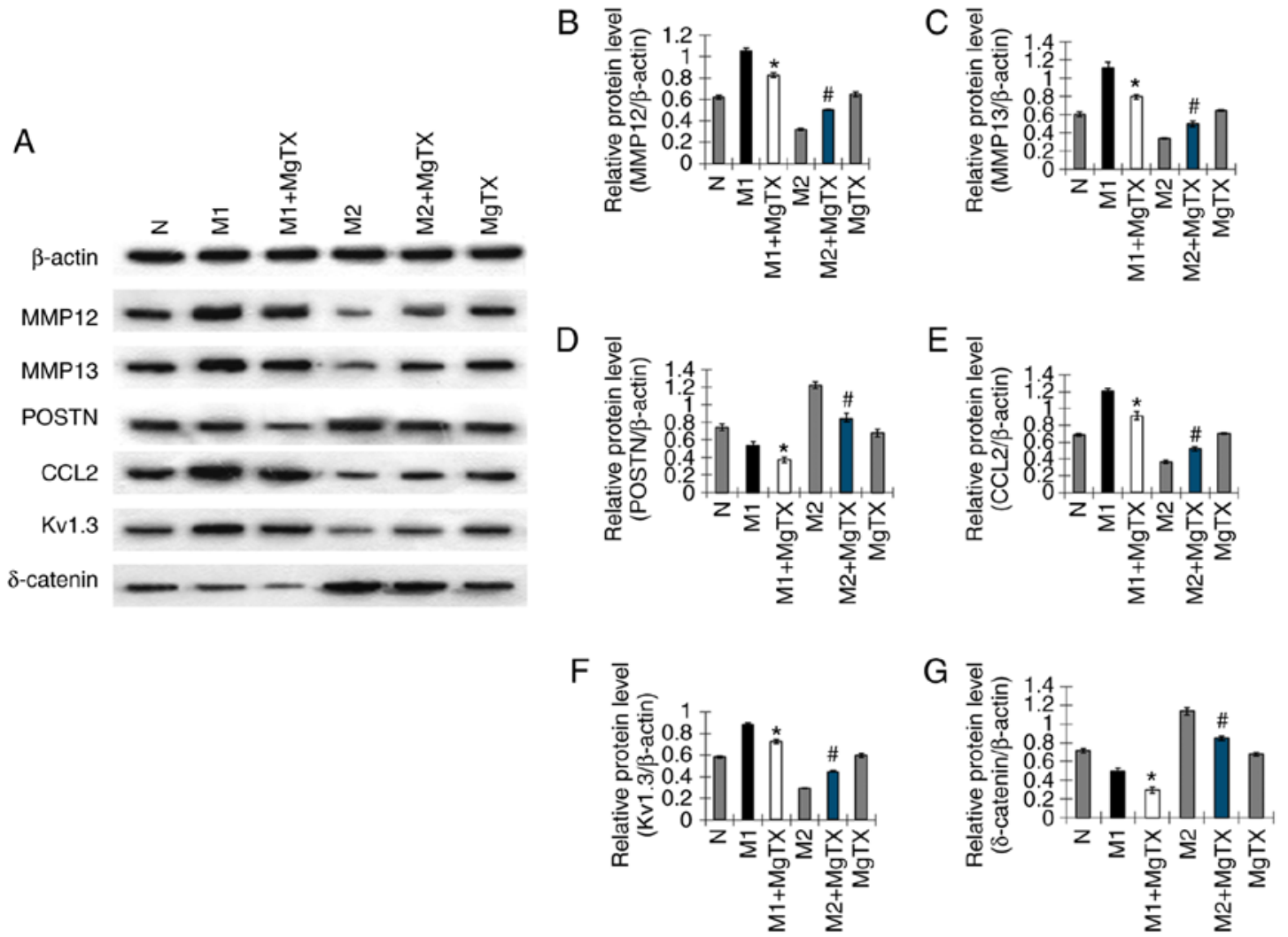

Figure 3. MgTX decreases the expression of HF-associated proteins in RAW264.7 cells. To confirm the results of HF-associated proteins from immunohistochemistry in RAW264.7 cells, (A) western blot analysis was performed to examine the expression of MMP12, MM13, POSTN, CCL2, Kv1.3 and $\delta$-catenin, which were downregulated by MgTX in the M1 phenotype groups. (B-G) MMP12, MM13, CCL2 and Kv1.3 were upregulated by MgTX in the M2 phenotype groups; however, POSTN and $\delta$-catenin were downregulated by MgTX in the M2 phenotype groups. No significant difference was observed between the control $(\mathrm{N})$ and MgTX groups. ${ }^{*} \mathrm{P}<0.05$ vs. $\mathrm{M} 1$ group; ${ }^{~} \mathrm{P}<0.05$ vs. M2 group. $\mathrm{n}=3$. MgTX, margatoxin; HF, hepatic fibrosis; MMP, matrix metalloproteinase; POSTN, periostin; CCL2, C-C motif chemokine ligand 2.

MgTX decreases the expression of HF-associated proteins in $R A W 264.7$ cells. The results of immunohistochemistry demonstrated that the aforementioned proteins were expressed in the liver tissue. Therefore, the current study aimed to determine protein expression in macrophages. The proteins associated with HF were determined in RAW264.7 cells treated with MgTX (10 nM; Control; M1, M1 + MgTX and M2, MgTX and M2 + MgTX), to examine whether macrophages are associated proteins that have been indicated to play a role in HF. This analysis was also performed to determine whether macrophages play a critical role in regulating the expression of proteins associated with HF. The levels of MMP12, MMP13, POSTN, CCL2, Kv1.3 and $\delta$-catenin were determined by western blot analysis. The results indicated that the levels of proteins associated with HF in the M1 phenotype macrophages treated with MgTX decreased compared with the other M1 phenotype groups. However, the levels of proteins associated with HF in the M2 phenotype macrophages treated with MgTX were downregulated or upregulated compared with the other M2 phenotype groups (Fig. 3).

MgTX regulates the secretion of cytokines in vivo. To explore the functional role of Kv1.3 in the regulation of cytokine secretion in CCl4-induced HF, ELISAs were performed to evaluate the circulation levels of inflammatory cytokines in the serum of mice with CCl4-induced HF. The results revealed that the serum levels of pro-inflammatory cytokines, including IL-1 $\beta$, IL-6, IL-20 and TNF- $\alpha$ were significantly decreased with MgTX treatment $(0.4179 \mathrm{mg} / \mathrm{kg})$, while the levels of anti-inflammatory cytokines, including IL-10, were increased in the mice with $\mathrm{CCl} 4$-induced HF treated with $\operatorname{MgTX}(0.4179 \mathrm{mg} / \mathrm{kg})$. However, TGF- $\beta$ expression was not significantly decreased in the CCL4 + MgTX treatment group compared with the model groups (Fig. 4).

MgTX regulates macrophage polarization in vitro. To examine whether MgTX can regulate macrophage polarization, M1 macrophages were induced using LPS $(1 \mu \mathrm{g} / \mathrm{ml})$ and IFN- $\gamma(10 \mathrm{ng} / \mathrm{ml})$ for $24 \mathrm{~h}$, and M2 macrophages were induced using IL-4 $(15 \mathrm{ng} / \mathrm{ml})$ for $24 \mathrm{~h}$. RT-qPCR indicated that treatment of M1 macrophages with MgTX (10 nM) decreased the mRNA expression of M1-related markers (iNOS, CCL2, TNF- $\alpha$ and IL-1 $\beta$ ), while the mRNA expression levels of M2-related markers (IL-10, CD163, Arg-1 and Mrc2) were upregulated by MgTX in M2 macrophages (Fig. 5A-H). The primer sequences are presented in Table I ( $\left.5^{\prime}-3^{\prime}\right)$. The results of western blot analysis were consistent with the results of RT-qPCR (Fig. 5I-Q). The results revealed that Kv1.3 contributed to the regulation of macrophage polarization.

Kv1.3 regulates macrophage polarization through the STATI/STAT6 pathway. The underlying mechanisms through which MgTX contributes to macrophage polarization was subsequently investigated. Previous reports have indicated that 

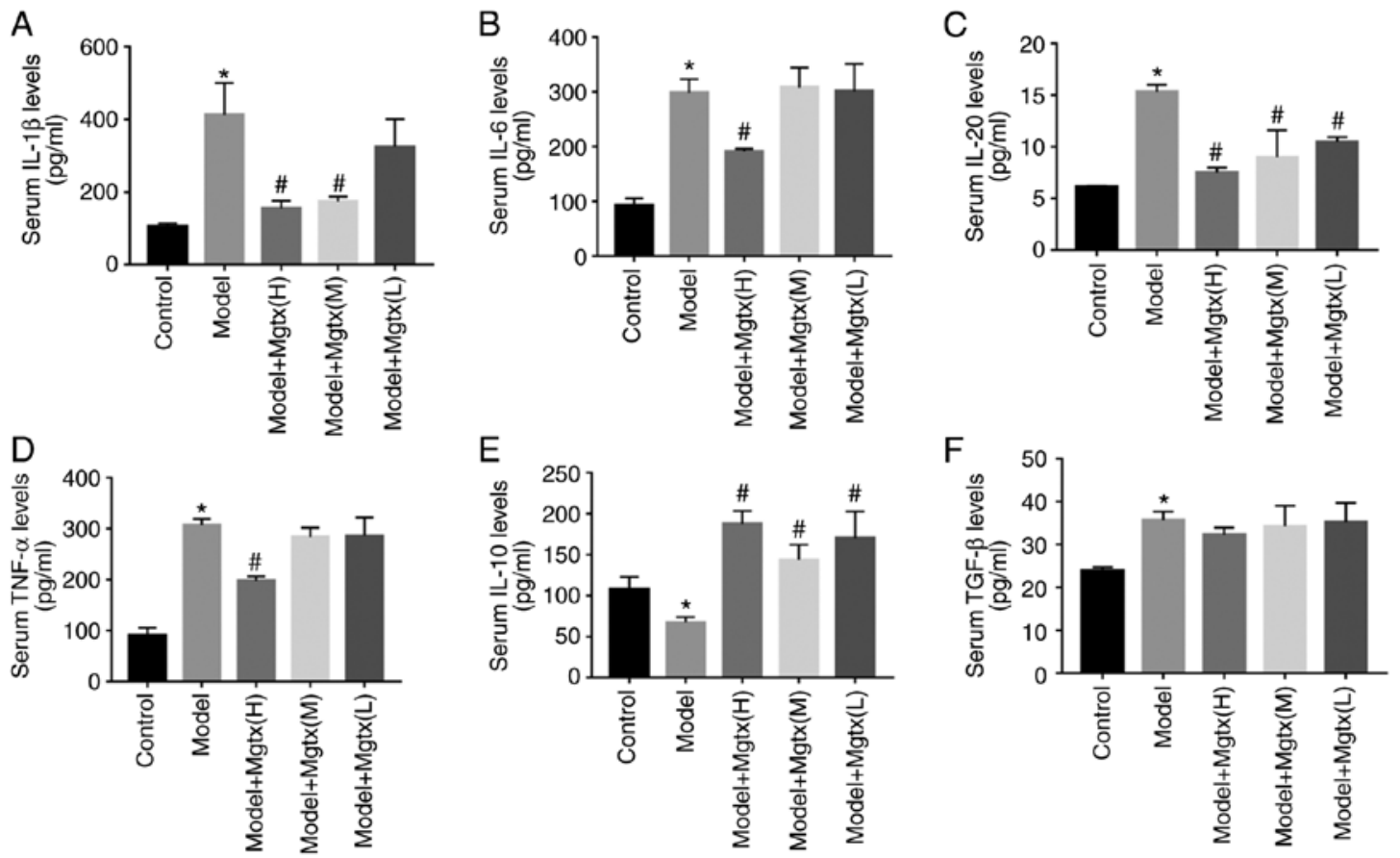

Figure 4. MgTX regulates cytokine secretion in vivo. A total of 3 different MgTX concentrations were injected into mice via intraperitoneal injection. The cytokines in mouse serum were measured using ELISA kits, $\operatorname{MgTX}(\mathrm{H}): 0.41 \mathrm{mg} / \mathrm{Kg}, \operatorname{MgTX}(\mathrm{M}): 0.14 \mathrm{mg} / \mathrm{Kg}, \mathrm{MgTX}(\mathrm{L}): 0.04 \mathrm{mg} / \mathrm{Kg}$. (A-F) ELISAs to detect the levels of pro-inflammatory cytokines (TNF- $\alpha$, IL-1 $\beta$, IL-6 and IL-20) in-mouse serum. Cytokine levels of IL-10 were inhibited compared with the HF model group, whereas these levels were increased compared with the HF model group treated with MgTX. The cytokine levels of TGF- $\beta$ group (n=6) increased in the model group, while there was no significant difference observed in the HF model group treated with MgTX. ${ }^{*} \mathrm{P}<0.05$ vs. control group; ${ }^{*} \mathrm{P}<0.05$ vs. model group. $n=6$. MgTX, margatoxin; TNF- $\alpha$, tumor necrosis factor- $\alpha$; IL, interleukin; TGF- $\beta$, transforming growth factor- $\beta$; HF, hepatic fibrosis.

the activation of STAT1 signaling pathways, by IFNs and/or TLR signaling, can switch macrophage toward the M1 phenotype, additionally, the activation of STAT6 by IL-4 and/or IL-13 has been demonstrated to switch macrophage function toward the M2 phenotype (29). In this study, the results of western blot analysis revealed that the phosphorylation of STAT1 was significantly increased in M1 macrophages, and was downregulated following $\operatorname{MgTX}(10 \mathrm{nM})$ treatment. Additionally, the phosphorylation of STAT6 was significantly increased in M2 macrophages, and was upregulated by MgTX treatment (Fig. 6). These results indicated that MgTX may regulate macrophage polarization through the STAT1/STAT6 signaling pathway.

\section{Discussion}

Fibrogenesis is a biochemical process that represents damage in the majority of chronic diseases of the liver. The activation of HSCs is an important pathophysiological mechanism in the initiation, progression and regression of liver fibrosis. A number of studies have identified crosstalk, which is at the level of hepatic microcirculation, between the HSCs, sinusoidal endothelial cells and KCs (30). Studies on animal models and human studies have indicated that HF is a reversible disease. The regression of HF can be possible in the early stages of the condition, and partial and prolonged recovery occurs in the late or advanced stages. The success of HF treatment can increase survival and decrease the occurrence of liver cirrhosis and hepatocellular carcinoma (31-36). Macrophages play a crucial role in the occurrence and resolution of liver fibrosis. It has been well established that infiltrating macrophages derived from monocytes and KCs play a key role in the initiation and resolution of $\mathrm{HF}$, and, in mouse models of chronic liver injury, it has been demonstrated that hepatic macrophages can promote the activation and survival of HSCs $(37,38)$. Furthermore, a subtype of restorative macrophages has been indicated to promote the resolution of fibrosis in chronic liver injury $(5,39)$. The current study demonstrated that MgTX can protect liver cells from CCl4-induced HF injury. The present study also explored whether MgTX attenuates liver fibrosis through the regulation of cytokine secretion and the polarization of macrophages.

The results of the current study indicated that MgTX decreased the expression of HF associated proteins in mice. Proteins of the MMP family are associated with the breakdown of the extracellular matrix (ECM) during physiological processes. MMP12 and MMP13 are important proteins that are associated with the ECM. Macrophages exhibit numerous functions in fibrogenesis, and specific subtypes promote fibrosis resolution through enhanced ECM degradation and by increasing MMP12 and MMP13 expression $(40,41)$. In the current study, the expression levels of MMP12 and MMP13 were increased in the HF model, and this was due to an excess of ECM that requires degradation. The results of the current study demonstrated that MMP12 and MMP13 expression decreased in CCl4-exposed mice treated with MgTX. POSTN is a $90-\mathrm{kDa}$ secretory matricellular protein that can regulate a number of effects during inflammation, wound healing and tissue injury, and regulates key molecules of the fibrotic matrix, including fibronectin and type I collagen via its EMILIN domain, to promote fibrosis degradation $(42,43)$. The results of the present study indicated that POSTN was significantly 

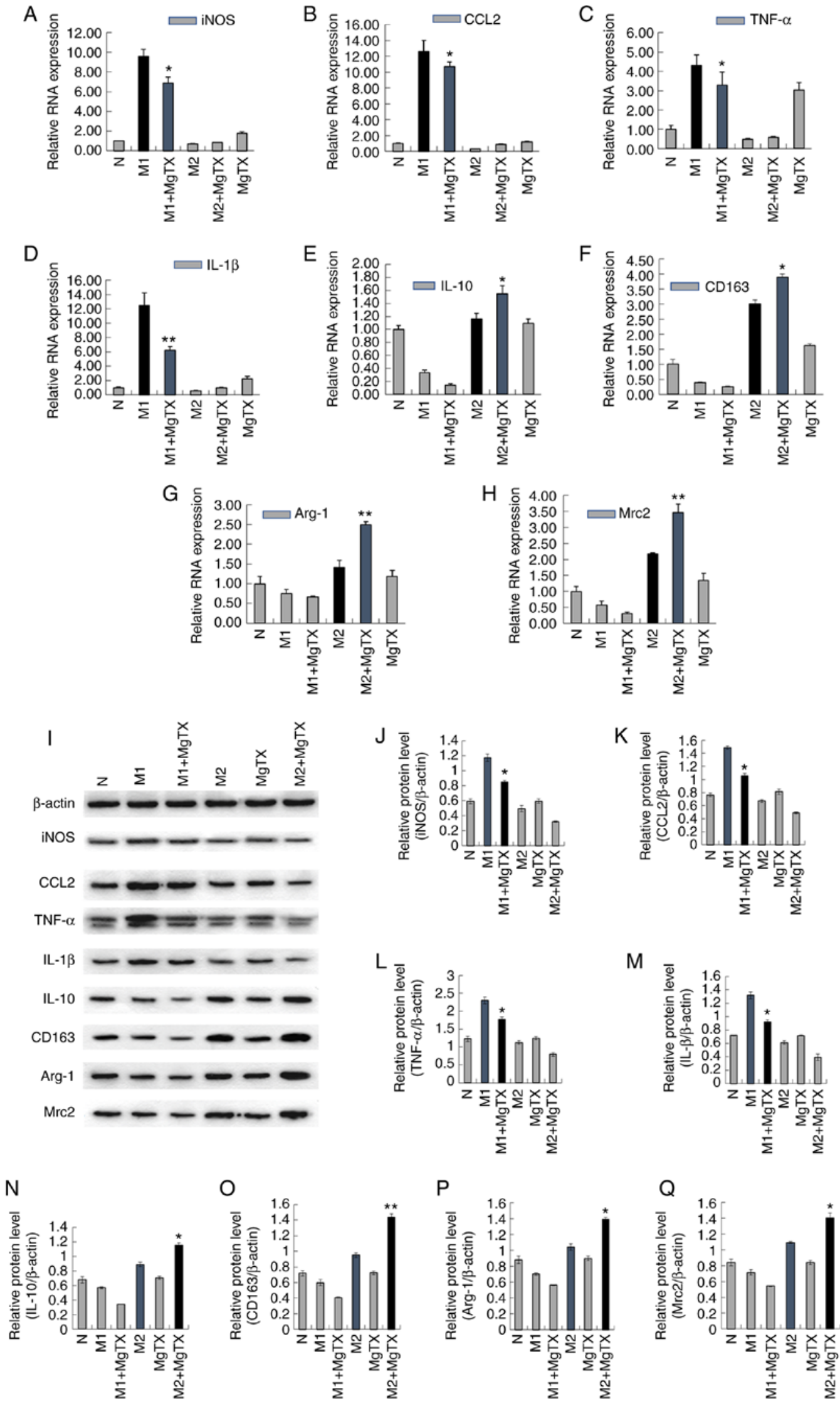

Figure 5. MgTX regulates macrophage polarization in vitro. Reverse transcription-quantitative PCR and western blot analysis were performed to detect the expression of M1 markers and M2 markers in RAW264.7 cells. (A-H) mRNA expression levels of iNOS, CCL2, TNF- $\alpha$ and IL-1 $\beta$ were downregulated by MgTX in M1 phenotype macrophages. mRNA expression levels of IL-10, CD163, Arg-1, MAC-2 were upregulated in M2 phenotype macrophages treated with MgTX compared with M2 phenotype macrophages. (I-Q) protein expression levels of M1 markers (iNOS, CCL2, TNF- $\alpha$ and IL-1 $\beta$ ) and protein expression levels of M2 markers (IL-10, CD163, Arg-1, Mrc2) were measured by western blot analysis. "P $<0.05,{ }^{* *} \mathrm{P}<0.01, \mathrm{M} 1$ vs. M1 + MgTX group, M2 vs. M2 + MgTX group. control group (N), $\mathrm{n}=3$. MgTX, margatoxin; CCL2, C-C motif chemokine ligand 2; TNF- $\alpha$, tumor necrosis factor- $\alpha$; IL, interleukin; CD, cluster of differentiation. 

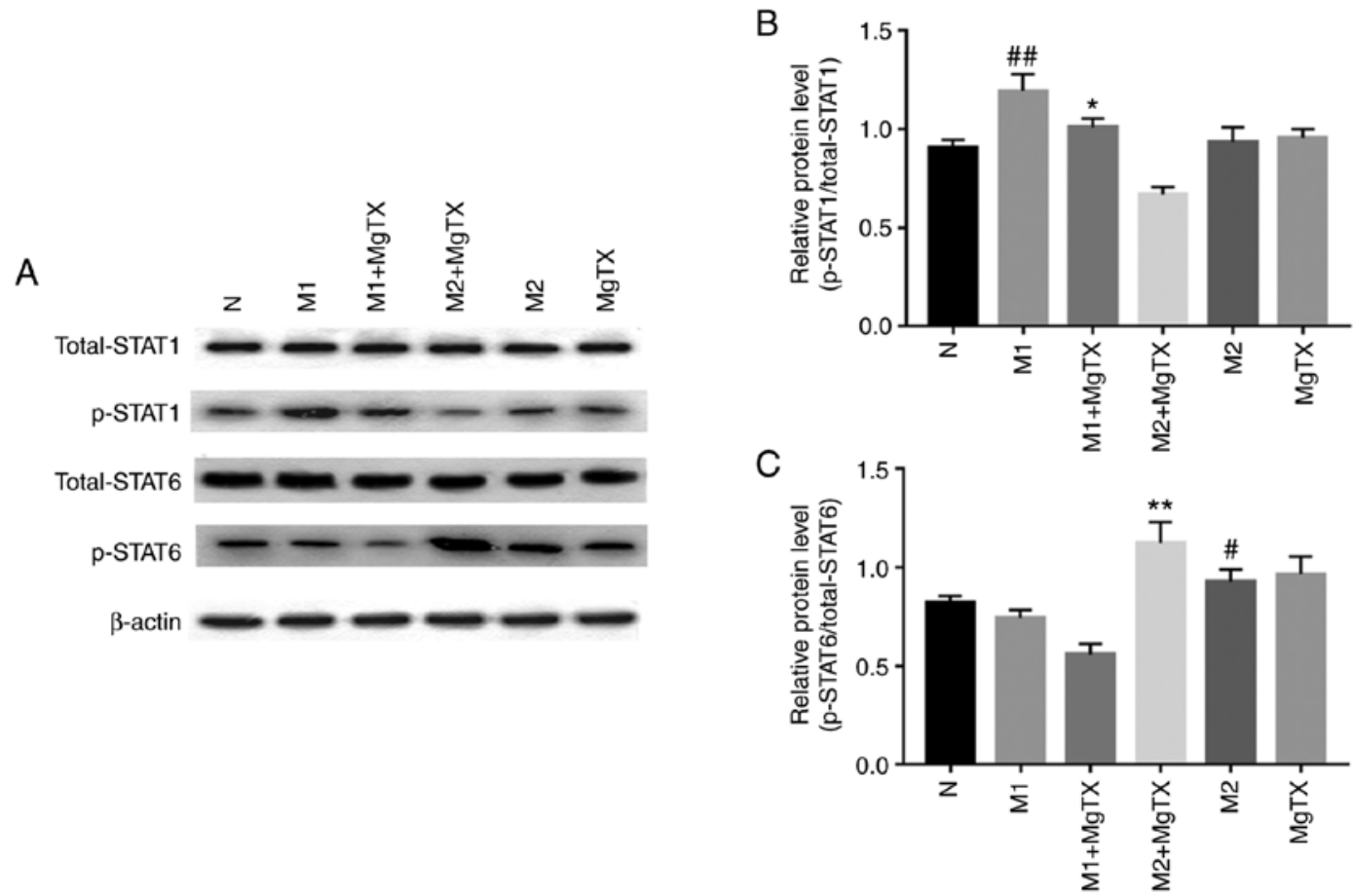

Figure 6. MgTX regulates macrophage polarization via the STAT1/STAT6 pathway. MgTX was administered to M1 phenotype macrophages or M2 phenotype macrophages, respectively, and the expression of phosphorylated STAT1/STAT6 was measured using western blot analysis. (A-C) Data are presented as the relative protein expression against control expression without treatment. The phosphorylated levels of STAT1/STAT6 in M1/M2 phenotype macrophages increased significantly compared with the control group, and p-STAT1 expression was downregulated in M1 phenotype macrophages treated with MgTX. ${ }^{*} \mathrm{P}<0.05$ vs. M1 group; ${ }^{\# \prime} \mathrm{P}<0.01$ vs. control group $(\mathrm{N})$. p-STAT6 was upregulated in M2 phenotype macrophages treated with MgTX. ${ }^{* *} \mathrm{P}<0.05$ vs. M2 group; ${ }^{\#} \mathrm{P}<0.05$ vs. control group. $\mathrm{n}=3$. MgTX, margatoxin.

downregulated by MgTX in the HF model compared with the model group. MMP12, MM13 and POSTN are associated with the progression of ECM and collagen. The results revealed that MgTX decreased the expression of MMP12, MMP13 and POSTN, in the CCl4-exposed group, attenuating HF. Exposure to $\mathrm{CCl} 4$ led to the elevation of liver enzymes, such as ALT and AST; however, there was no significant change in the liver fibrosis model with MgTX treatment. Additionally, a number of studies have indicated that the M1 phenotype cells play an important role in promoting $\operatorname{HF}(29,44)$. In this study, in RAW264.7 cell lines, the expression of proteins associated with HF were decreased in M1 phenotype macrophages treated with MgTX, which exerts an anti-fibrotic effect in the progressive stage.

A therapeutic strategy for the treatment of $\mathrm{HF}$ is to inhibit inflammatory monocyte infiltration. Kv1.3 plays a critical role in monocyte migration, and the inhibition of Kv1.3 can inhibit monocyte chemotaxis and monocyte infiltration into injury brain (45). In the current study, Kv1.3 was demonstrated to be associated with macrophage migration in acute liver injury; Kv1.3 was indicated to downregulate the expression of $\delta$-catenin to inhibit macrophage migration. According to another study by our group (unpublished data), RNA-seq profiling analysis revealed that MgTX treatment downregulated $\delta$-catenin. According to wound healing and Transwell assays, $\delta$-catenin overexpression in RAW264.7 cells promoted migration, which was suppressed upon silencing of $\delta$-catenin. Another study revealed that monocyte recruitment following light-induced cell death was associated with the spatial-temporal expression of CCL2, and the suppression of CCL2 in Müller cells decreased the recruitment of monocytes/microglia (46). Additionally, previous studies have revealed that macrophages may promote HSC migration via CCL2 secretion, which can regulate the crosstalk between cholangiocytes and portal fibroblasts $(47,48)$. The inhibition of macrophage migration infilter into the liver can reduce the immunological reaction; thus, in this study a decrease in the expression of Kv1.3, $\delta$-catenin and CCL2 in the mice with CCl4-induced injury treated with MgTX was observed. On the whole, the current study indicated that MgTX inhibited macrophage migration to attenuate $\mathrm{HF}$ in mice.

HF typically results from an inflammatory process that affects hepatocytes or biliary cells. Inflammation leads to the activation of effector cells, which results in the deposition of ECM. Cytokines are released from inflammatory cells and play an important role in HF as a key effector. The TGF- $\beta$ cascade plays a major role in fibrogenesis due to TGF- $\beta$ being a potent stimulator of the synthesis of extracellular matrix proteins (49). Pro-inflammatory cytokines, including IL-6, IL-1 $\beta$ and TNF- $\alpha$, are released from innate immune cells and promote fibrogenesis via active HSCs. A recent study demonstrated that the treatment of mice with recombinant IL-20 increased the expression of the pro-fibrotic cytokines, TGF- $\beta$ and TNF- $\alpha$, and enhanced collagen synthesis in the liver; treatment with neutralizing antibodies against IL-20 or IL20RA was revealed to ameliorate the CCl4-induced HF in mice (50). Cytokines of the IL-10 family exert protective effects, and IL-10 has been identified to exhibit a suppressive effect, which inhibits the innate and adaptive immune responses and prevents increased exacerbations. Therefore, 
IL-10 plays a protective role in the prevention of tissue injury in chronic fibroproliferative diseases. Additionally, this has been demonstrated in wound healing assays of scar formation in IL-10 knockout mice (51). The results of the current study demonstrated that MgTX decreased the serum levels of IL-6, IL-1 $\beta$, IL-20 and TNF- $\alpha$, increased the IL-10 serum levels, but did not affect the TGF- $\beta$ levels significantly. These results indicated that MgTX regulated cytokine secretion in macrophages; however, this effect was decreased in HSCs. Thus, HF can be attenuated by MgTX through the regulation of the serum levels of a number of cytokines.

A variety of macrophage subtypes release a number of cytokines and exhibit functions in the pathophysiological process. The M1 and M2 phenotype polarization of macrophages may directly influence the outcome in chronic hepatic injury (52). In this study, RT-qPCR and western blot analysis indicated that the levels of M1-related markers were downregulated by MgTX, and that the levels of M2-related markers were upregulated by MgTX. A number of studies have indicated that STATs are crucial factors in M1 and M2 macrophage polarization $(53,54)$. p-STAT1 is required to promote M1 type macrophage activation in the presence of IFN- $\gamma$ from innate lymphocytes (55). By contrast, p-STAT6 is an important regulator of M2 type macrophage polarization in the presence of IL-13 or IL-4 during the TH2 cell-mediated immune responses $(56,57)$. Therefore, the current study examined the effects of MgTX on STAT signaling during macrophage polarization. The phosphorylation and protein expression of p-STAT1 was markedly decreased in M1 macrophages treated with MgTX, and the phosphorylation levels of p-STAT6 were increased in M2 macrophages treated with MgTX. Therefore, MgTX was indicated to regulate macrophage polarization via the STAT signaling pathway. Furthermore, the phenotype of macrophages can be switched by MgTX; thus, it would be of interest to examine the migratory ability of macrophages in future studies.

$\mathrm{HF}$, which is caused by a number of etiologies, is a common pathological characteristic of end-stage hepatic diseases. Advanced fibrosis can lead to cirrhosis, and currently, there is no effective anti-fibrotic treatment that has been approved for human use, at least to the best of our knowledge. Thus, the identification of novel therapeutic options with which to prevent the progression of liver fibrosis is urgently required. The current study demonstrated that MgTX can ameliorate HF by regulating macrophage polarization and cytokine secretion. These results provide novel information on treatment options for the prevention and reversion of liver fibrosis. However, further studies are required to examine whether MgTX may be an effective candidate for the treatment of advanced-stage fibrosis or cirrhosis and whether MgTX may be a novel candidate for the treatment of HF or other immunological diseases.

\section{Acknowledgements}

Not applicable.

\section{Funding}

The current study was supported by a grant from the National Natural Science Foundation of China (grant no. 81500473).

\section{Availability of data and materials}

All data generated or analyzed during this study are included in this published article or are available from the corresponding author on reasonable request.

\section{Authors' contributions}

$\mathrm{JL}$ and YHL conceived and designed the experiments. BMW and JDL performed the experiments. BMW analyzed the data and wrote the paper. All authors have read and approved the final manuscript.

\section{Ethics approval and consent to participate}

All animal protocols were approved by the Animal Care and Use Committee of Anhui Medical University, China.

\section{Patient consent for publication}

Not applicable.

\section{Competing interests}

The authors declare that they have no competing interests.

\section{References}

1. Mederacke I, Hsu CC, Troeger JS, Huebener P, Mu X, Dapito DH, Pradere JP and Schwabe RF: Fate tracing reveals hepatic stellate cells as dominant contributors to liver fibrosis independent of its aetiology. Nat Commun 4: 2823, 2013.

2. Zheng Z, Xu X, Zhang X, Wang A, Zhang C, Hüttemann M, GrossmanLI,ChenLC,RajagopalanS,SunQ andZhang K:Exposure to ambient particulate matter induces a NASH-like phenotype and impairs hepatic glucosemetabolismin an animal model.JHepatol58: 148-154, 2013.

3. Czaja AJ and Carpenter HA: Progressive fibrosis during corticosteroid therapy of autoimmune hepatitis. Hepatology 39: 1631-1638, 2004.

4. Schuppan D and Kim YO: Evolving therapies for liver fibrosis. J Clin Invest 123: 1887-1901, 2013.

5. Duffield JS, Forbes SJ, Constandinou CM, Clay S, Partolina M, Vuthoori S, Wu S, Lang R and Iredale JP: Selective depletion of macrophages reveals distinct, opposing roles during liver injury and repair. J Clin Invest 115: 56-65, 2005.

6. Ramachandran P and Iredale JP: Macrophages: Central regulators of hepatic fibrogenesis and fibrosis resolution. J Hepatol 56: 1417-1419, 2012.

7. Chawla A, Nguyen KD and Goh YP: Macrophage-mediated inflammation in metabolic disease. Nat Rev Immunol 11: 738-749, 2011.

8. Mosser DM and Edwards JP: Exploring the full spectrum of macrophage activation. Nat Rev Immunol 8: 958-969, 2008.

9. Gundra UM, Girgis NM, Gonzalez MA, San Tang M, Van Der Zande HJP, Lin JD, Ouimet M, Ma LJ, Poles J, Vozhilla N, et al: Vitamin A mediates conversion of monocyte-derived macrophages into tissue-resident macrophages during alternative activation. Nat Immunol 18: 642-653, 2017.

10. Borthwick LA, Suwara MI, Carnell SC, Green NJ, Mahida R, Dixon D, Gillespie CS, Cartwright TN, Horabin J, Walker A, et al: Pseudomonas aeruginosa induced airway epithelial injury drives fibroblast activation: A mechanism in chronic lung allograft dysfunction. Am J Transplant 16: 1751-1765, 2016.

11. Gordon S and Martinez FO: Alternative activation of macrophages: Mechanism and functions. Immunity 32: 593-604, 2010.

12. Murray PJ, Allen JE, Biswas SK, Fisher EA, Gilroy DW, Goerdt S, Gordon S, Hamilton JA, Ivashkiv LB, Lawrence T, et al: Macrophage activation and polarization: Nomenclature and experimental guidelines. Immunity 41: 14-20, 2014. 
13. Sica A, Invernizzi P and Mantovani A: Macrophage plasticity and polarization in liver homeostasis and pathology. Hepatology 59: 2034-2042, 2014.

14. Heymann F, Trautwein C and Tacke F: Monocytes and macrophages as cellular targets in liver fibrosis. Inflamm Allergy Drug Targets 8: 307-318, 2009.

15. Murray PJ and Wynn TA: Protective and pathogenic functions of macrophage subsets. Nat Rev Immunol 11: 723-737, 2011.

16. Chiaramonte MG, Donaldson DD, Cheever AW and Wynn TA: An IL-13 inhibitor blocks the development of hepatic fibrosis during a T-helper type 2-dominated inflammatory response. J Clin Invest 104: 777-785, 1999.

17. Kaviratne M, Hesse M, Leusink M, Cheever AW, Davies SJ, McKerrow JH, Wakefield LM, Letterio JJ and Wynn TA: IL-13 activates a mechanism of tissue fibrosis that is completely TGF-beta independent. J Immunol 173: 4020-4029, 2004.

18. McKenzie GJ, Bancroft A, Grencis RK and McKenzie AN: A distinct role for interleukin-13 in Th2-cell-mediated immune responses. Curr Biol 8: 339-342, 1998

19. McKenzie GJ, Fallon PG, Emson CL, Grencis RK and McKenzie AN: Simultaneous disruption of interleukin (IL)-4 and IL-13 defines individual roles in T helper cell type 2-mediated responses. J Exp Med 189: 1565-1572, 1999.

20. Mackenzie AB, Chirakkal H and North RA: Kv1.3 potassium channels in human alveolar macrophages. Am J Physiol Lung Cell Mol Physiol 285: L862-L868, 2003.

21. KazamaI,Maruyama Y,Murata Y and Sano M: Voltage-dependent biphasic effects of chloroquine on delayed rectifier $\mathrm{K}(+)$-channel currents in murine thymocytes. J Physiol Sci 62: 267-274, 2012.

22. Toldi G, Bajnok A, Dobi D, Kaposi A, Kovács L, Vásárhelyi B and Balog A: The effects of Kv1.3 and IKCa1 potassium channel inhibition on calcium influx of human peripheral $\mathrm{T}$ lymphocytes in rheumatoid arthritis. Immunobiology 218 : 311-316, 2013.

23. Beeton C, Wulff H, Standifer NE, Azam P, Mullen KM, Pennington MW, Kolski-Andreaco A, Wei E, Grino A, Counts DR, et al: Kv1.3 channels are a therapeutic target for T cell-mediated autoimmune diseases. Proc Natl Acad Sci USA 103: 17414-17419, 2006

24. Chi V, Pennington MW, Norton RS, Tarcha EJ, Londono LM, Sims-Fahey B, Upadhyay SK, Lakey JT, Iadonato S, Wulff $\mathrm{H}$, et al: Development of a sea anemone toxin as an immunomodulator for therapy of autoimmune diseases. Toxicon 59: 529-546. 2012

25. Kazama I, Maruyama Y, Endo Y, Toyama H, Ejima Y, Matsubara $M$ and Kurosawa S: Overexpression of delayed rectifier $\mathrm{K}(+)$ channels promotes in situ proliferation of leukocytes in rat kidneys with advanced chronic renal failure. Int $J$ Nephrol 2012,581581, 2012.

26. Shao PP, Liu CJ, Xu Q, Zhang B, Li SH, Wu Y, Sun Z and Cheng LF: Eplerenone reverses cardiac fibrosis via the suppression of tregs by inhibition of Kv1.3 channel. Front Physiol 9: 899, 2018.

27. Moreno C, Prieto P, Macías Á, Pimentel-Santillana M, de la Cruz A, Través PG, Boscá L and Valenzuela C: Modulation of voltage-dependent and inward rectifier potassium channels by 15-epi-lipoxin-A4 in activated murine macrophages: Implications in innate immunity. J Immunol 191: 6136-6146, 2003.

28. Livak KJ and Schmittgen TD: Analysis of relative gene expression data using real-time quantitative PCR and the 2(-Delta Delta C(T)) method. Methods 25: 402-408, 2001

29. Yang Y, Wu XQ, Li WX, Huang HM, Li HD, Pan XY, Li XF, Huang C, Meng XM, Zhang L, et al: PSTPIP2 connects DNA methylation to macrophage polarization in CCL4-induced mouse model of hepatic fibrosis. Oncogene 37: 6119-6135, 2018.

30. Bitto N, Liguori E and La Mura V: Coagulation, microenvironment and liver fibrosis. Cells 7: pii: E85, 2018.

31. Wang KK and Czaja AJ: Hepatocellular carcinoma in corticosteroid-treated severe autoimmune chronic active hepatitis. Hepatology 8: 1679-1683, 1988.

32. Nishiguchi S, Kuroki T, Nakatani S, Morimoto H, Takeda T, Nakajima S, Shiomi S, Seki S, Kobayashi K and Otani S: Randomised trial of effects of interferon-alpha on incidence of hepatocellular carcinoma in chronic active hepatitis $\mathrm{C}$ with cirrhosis. Lancet 346: 1051-1055, 1995.

33. Roberts SK, Therneau TM and Czaja AJ: Prognosis of histological cirrhosis in type 1 autoimmune hepatitis. Gastroenterology 110: $848-857,1996$.
34. Singal AG, Volk ML, Jensen D, Di Bisceglie AM and Schoenfeld PS: A sustained viral response is associated with reduced liver-related morbidity and mortality in patients with hepatitis C virus. Clin Gastroenterol Hepatol 8: 280-288, 288.e1, 2010.

35. Lok AS, Everhart JE, Wright EC, Di Bisceglie AM, Kim HY, Sterling RK, Everson GT, Lindsay KL, Lee WM, Bonkovsky HL, et al: Maintenance peginterferon therapy and other factors associated with hepatocellular carcinoma in patients with advanced hepatitis C. Gastroenterology 140: 840-849, 2011

36. Bruix J, Poynard T, Colombo M, Schiff E, Burak K, Heathcote EJ, Berg T, Poo JL, Mello CB, Guenther R, et al: Maintenance therapy with peginterferon alfa- $2 \mathrm{~b}$ does not prevent hepatocellular carcinoma in cirrhotic patients with chronic hepatitis $\mathrm{C}$. Gastroenterology 140: 1990-1999, 2011.

37. Krenkel $\mathrm{O}$ and Tacke F: Liver macrophages in tissue homeostasis and disease. Nat Rev Immunol 17: 306-321, 2017.

38. Seki E and Schwabe RF: Hepatic inflammation and fibrosis: Functional links and key pathways. Hepatology 61: 1066-1079, 2015.

39. Baeck C, Wei X, Bartneck M, Fech V, Heymann F, Gassler N, Hittatiya K, Eulberg D, Luedde T, Trautwein C and Tacke F: Pharmacological inhibition of the chemokine $\mathrm{C}-\mathrm{C}$ motif chemokine ligand 2 (monocyte chemoattractant protein 1) accelerates liver fibrosis regression by suppressing $\mathrm{Ly}-6 \mathrm{C}(+)$ macrophage infiltration in mice. Hepatology 59: 1060-1072, 2014.

40. Ramachandran P, Pellicoro A, Vernon MA, Boulter L, Aucott RL, Ali A, Hartland SN, Snowdon VK, Cappon A, Gordon-Walker TT, et al: Differential Ly-6C expression identifies the recruited macrophage phenotype, which orchestrates the regression of murine liver fibrosis. Proc Natl Acad Sci USA 109: E3186-E3195, 2012.

41. Madala SK, Pesce JT, Ramalingam TR, Wilson MS, Minnicozzi S, Cheever AW, Thompson RW, Mentink-Kane MM and Wynn TA: Matrix metalloproteinase 12-deficiency augments extracellular matrix degrading metalloproteinases and attenuates IL-13-dependent fibrosis. J Immunol 184 : 3955-3963, 2010.

42. Conway SJ, Izuhara K, Kudo Y, Litvin J, Markwald R, Ouyang G, Arron JR, Holweg CT and Kudo A: The role of periostin in tissue remodeling across health and disease. Cell Mol Life Sci 71: 1279-1288, 2014

43. Mosher DF, Johansson MW, Gillis ME and Annis DS: Periostin and TGF- $\beta$-induced protein: Two peas in a pod? Crit Rev Biochem Mol Biol 50: 427-439, 2015.

44. Zhao XA, Chen G, Liu Y, Chen Y, Wu H, Xiong Y, Wang G, Jia B, Li Y, Xia J, et al: Curcumin reduces Ly6Chi monocyte infiltration to protect against liver fibrosis by inhibiting Kupffer cells activation to reduce chemokines secretion. Biomed Pharmacother 106: 868-878, 2018

45. Eder C: Ion channels in monocytes and microglia/brain macrophages: Promising therapeutic targets for neurological diseases. J Neuroimmunol 224: 51-55, 2010.

46. Rutar M, Natoli $R$ and Provis JM: Small interfering RNA-mediated suppression of Ccl2 in Müller cells attenuates microglial recruitment and photoreceptor death following retinal degeneration. J Neuroinflammation 9: 221, 2012.

47. Pellicoro A, Ramachandran P, Iredale JP and Fallowfield JA: Liver fibrosis and repair: Immune regulation of wound healing in a solid organ. Nat Rev Immunol 14: 181-194, 2014.

48. Kruglov EA, Nathanson RA, Nguyen T and Dranoff JA: Secretion of MCP-1/CCL2 by bile duct epithelia induces myofibroblastic transdifferentiation of portal fibroblasts. Am J Physiol Gastrointest Liver Physiol 290: G765-G771, 2006.

49. Rockey DC, Bell PD and Hill JA: Fibrosis-a common pathway to organ injury and failure. N Engl J Med 372: 1138-1149, 2015.

50. Chiu YS, Wei CC, Lin YJ, Hsu YH and Chang MS: IL-20 and IL-20R1 antibodies protect against liver fibrosis. Hepatology 60: 1003-1014, 2014

51. Sziksz E, Pap D, Lippai R, Béres NJ, Fekete A, Szabó AJ and Vannay Á: Fibrosis related inflammatory mediators: Role of the IL-10 cytokine family. Mediators Inflamm 2015: 764641, 2015.

52. Galastri S, Zamara E, Milani S, Novo E, Provenzano A, Delogu W, Vizzutti F, Sutti S, Locatelli I, Navari N, et al: Lack of CC chemokine ligand 2 differentially affects inflammation and fibrosis according to the genetic background in a murine model of steatohepatitis. Clin Sci (Lond) 123: 459-471, 2012. 
53. Khan Z, Cao DY, Giani JF, Bernstein EA, Veiras LC, Fuchs S, Wang Y, Peng Z, Kalkum M, Liu GY and Bernstein KE: Overexpression of the $\mathrm{C}$-domain of angiotensin-converting enzyme reduces melanoma growth by stimulating M1 macrophage polarization. J Biol Chem 294: 4368-4380, 2019.

54. Ding N, Wang Y, Dou C, Liu F, Guan G, Wei K, Yang J, Yang M, Tan J, Zeng W and Zhu C: Physalin D regulates macrophage M1/M2 polarization via the STAT1/6 pathway. J Cell Physiol 234: 8788-8796, 2019.

55. Darnell JE Jr, Kerr IM and Stark GR: Jak-STAT pathways and transcriptional activation in response to IFNs and other extracellular signaling proteins. Science 264: 1415-1121, 1994.

56. Herbert DR, Hölscher C, Mohrs M, Arendse B, Schwegmann A, Radwanska M, Leeto M, Kirsch R, Hall P, Mossmann H, et al: Alternative macrophage activation is essential for survival during schistosomiasis and downmodulates T helper 1 responses and immunopathology. Immunity 20: 623-635, 2004.
57. BrombacherF, Arendse B,Peterson R,Hölscher A and Hölscher C: Analyzing classical and alternative macrophage activation in macrophage/neutrophil-specific IL-4 receptor-alpha-deficient mice. Methods Mol Biol 531: 225-252, 2009.

(i) 1 This work is licensed under a Creative Common Attribution-NonCommercial-NoDerivatives 4.0 International (CC BY-NC-ND 4.0) License. 